\title{
Mauvaises herbes et graminées à Houailou (Nouvelle-Calédonie)
}

Jacqueline de La Fontinelle

\section{(2) OpenEdition \\ 1 Journals}

Édition électronique

URL : http://journals.openedition.org/jso/1370

DOI : $10.4000 /$ jso. 1370

ISSN : 1760-7256

\section{Éditeur}

Société des océanistes

\section{Édition imprimée}

Date de publication : 1 décembre 2002

Pagination : 39-43

ISSN : 0300-953x

\section{Référence électronique}

Jacqueline de La Fontinelle, « Mauvaises herbes et graminées à Houailou (Nouvelle-Calédonie) », Journal de la Société des Océanistes [En ligne], 114-115 | Année 2002, mis en ligne le 26 mai 2008, consulté le 22 mars 2021. URL : http://journals.openedition.org/jso/1370 ; DOl : https://doi.org/ $10.4000 /$ jso. 1370

\section{(c) (1) (9)}

Journal de la société des océanistes est mis à disposition selon les termes de la Licence Creative Commons Attribution - Pas d'Utilisation Commerciale - Pas de Modification 4.0 International. 


\section{Mauvaises herbes et graminées à Houailou (Nouvelle-Calédonie)}

par

Jacqueline de LA FONTINELLE *

\section{RÉSUMÉ}

La classification ajië (Houailou, NouvelleCalédonie) montre que les graminées ont été repérées et identifiées comme constituant une famille distincte des autres " herbes". D'un usage constant et très important tant dans la vie courante que lors des cérémonies de deuil - nattes, liens, nouds de toutes sortes (portage, interdits, calendriers, chaumes ou signes de jachères...) -, les graminées ne sont pourtant jamais entrées en culture.

Mots-CLÉS : Nouvelle-Calédonie, Houailou, graminées, nattes, chaume, interdits.

Je me souviens d'un jour ensoleillé de 1968 où Jacques et moi admirions au-dessus de Nessakoya les gradins bleus du ciel et des Ageratum envahissant les dernières tarodières. Nous discutions à bâtons rompus des idéologies qui s'affrontaient en France et des cultures vivrières en Nouvelle-Calédonie. L'idée alors nouvelle d'A.-G. Haudricourt sur l'origine de la mise en culture du riz - « mauvaise herbe » spontanée des tarodières ensuite abandonnées - l'enthousiasmait. Ces quelques lignes pourraient être la continuation de cette conversation lointaine.

Nous présenterons successivement les herbes dewi et les graminées $j a ̂$ puis nous tenterons de montrer ce que furent leurs utilisations, et ce qui reste de celles-ci. Nous évoquerons aussi les

\begin{abstract}
Graminae have been classified apart from weeds in the Ajië language (Houailou, New Caledonia). Their uses thatch, mats, threads and strings as well as knots of every kind to carry loads or prohibit picking fruit, trodding on a place abandoned after the death of a man, made them constantly present in every day life and during ceremonies yet graminae have not been cultivated as food crop.
\end{abstract}

Keywords : New Caledonia, Houailou, GraminaCEAE, PLAITS, THATCH, TABOOS.

valeurs symboliques liées à leurs usages. Cela nous conduira à donner quelques indications sur des techniques abandonnées et sur la place imprécise des graminées dans la classification traditionnelle "plante femelle versus plante mâle ». Nous conclurons en présentant la notion jâ dont le champ sémantique couvre la production spontanée de « fibres » — liens et nœuds —, et le lien entre « signe d'interdit » et « marque de la mort».

L'herbe occupe, ou a occupé, dans nos représentations une place que l'on peut penser modeste mais qui s'avère plus importante que l'on pourrait croire dès que l'on s'y intéresse : " mauvaises herbes», " herbes de la SaintJean », « herbes » culinaires..., sans compter le

* Professeur émérite à l'Institut national des langues et civilisations orientales, Paris. 
double héritage contradictoire d'images bibliques de «l'herbe folle » que le soleil dessèche et les visions des riches prairies qui engraissent le bétail...

L'étude des termes ajië ${ }^{1}$ qui désignent "l'herbe » et « les herbes » montre que ces végétaux ont joué un grand rôle utilitaire et symbolique. Il n'en est plus ainsi : les techniques abandonnées ont fait oublier le vocabulaire qui les accompagnait - mais beaucoup de croyances relatives à la jachère et à la mort perdurent.

\section{Dewi et nédewi, « l'herbe et la brousse »}

Dewi est le terme générique correspondant à notre mot « herbe ». Dewi désigne aussi bien les " mauvaises herbes », celles qui envahissent les jardins et les billons d'ignames et sur lesquelles se posent volontiers les maléfices du "mauvais œil », que les herbes salutaires de la pharmacopée. Comme pour nous, l'herbe a des connotations positives et négatives ${ }^{2}$.

Les noms des plantes médicinales devaient rester secrets ; les feuilles, inflorescences, écorces, etc. sont désignées par le terme dewi par « respect » car en fait, leur vertu appartient à des familles et ne peut se manifester qu'en leurs soins. Dewi masque donc le nom des plantes, mais il est aussi le signe d'un langage, le dö mêrêa, le "vrai langage ", généralement oublié aujourd'hui, fait d'évitements et de métaphores, apanage des gens importants, les "vieux ». Le soigneur mâchait les herbes et les crachotait en suivant d'invisibles trajets sur le corps du patient. Était-ce les méridiens de la science chinoise? Malheureusement ces pratiques n'ont pas été étudiées quand elles étaient encore vivantes.

Nédewi ${ }^{3}$ évoque "la brousse », les zones déboisées retournées à l'état sauvage, là où tout est possible, le meilleur comme le pire, car s'y rencontraient les esprits des morts, les kavere ${ }^{4}$ et les enfants hamadryades qui attendaient d'être adoptés. C'est là aussi, dans les jachères, que l'on pouvait récolter en temps de disette, les subspontanées qui permettaient de survivre.

\section{Les graminées jâ}

Maurice Leenhardt, dans son Vocabulaire et grammaire de la langue de Houailou, avait écrit : «jâ, préfixe collectif dans la composition de divers noms de plantes dont les fibres conviennent à la composition de nattes » (1935:110).

En fait, à part une exception dont nous reparlerons plus loin, le recensement botanique des graminées autochtones correspond très régulièrement aux « herbes" dont le nom a pour première syllabe $j \hat{a}$, sorte de classificateur. Les graminées sont donc bien différenciées des autres plantes qui couvrent le sol et, pourtant, aucune n'est entrée en culture pour ses graines. Est-ce la concurrence des tubercules (taros, ignames) qui ne l'a pas permise?

\section{Les chaumes dèo et mémé}

Deux graminées sont citées souvent sans leur préfixe indicatif (mais, à l'enquête, elles étaient classées avec les autres $j \hat{a}-$-). Il s'agit de dèo et de mémé. Il est vrai que leur utilisation, et par suite le rôle symbolique qui leur été conféré, les distinguaient des autres graminées.

Dèo, Imperata cylindrica (L.) Beauv., Graminée, est inséparable de la vision des collines blanchies par ses milliers d'épis en fleurs que le vent fait onduler comme les eaux agitées 5 . Elle symbolisait la paix, l'époque où les hommes pou-

1. Ajië est le nom de la langue parlée dans la région de Houailou-Poya. Cette langue ainsi que ces dialectes fait partie du groupe central des langues kanak de Nouvelle-Calédonie. La graphie n'est toujours pas fixée officiellement pour cette langue qui figure parmi les langues retenues au baccalauréat. Comme toute langue orale, l'ajië présente de nombreuses formes de parlers. La prononciation de la vallée diffère de celle de la côte en ce qui concerne la longueur vocalique (par exemple, on prononce dewi

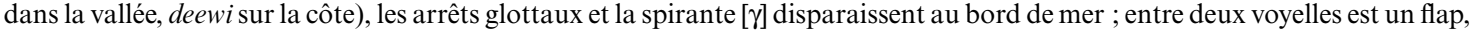
oral entre voyelles orales, nasal entre voyelles nasales et $<\hat{\mathrm{u}}>$ représente la voyelle nasale postérieure étirée de premier degré d'aperture. Les locuteurs s'entendaient pour dire que la langue parlée à Poya (côte ouest) était « la plus pure ». La notation du dictionnaire de la Felp (2001) correspond aux réalisations des gens du bord de mer tandis que les notations utilisées dans cet article rendent compte de la prononciation des gens de la vallée de Houailou. Il existe aussi des différences de prononciation entre les générations.

2. Ni le français, ni l'ajië ne possèdent de termes spécifiques, alors que l'anglais distingue « grass », de « weeds » ou encore d'« herbs".

3. Dans nédewi, né est un morphème qui indique que le terme est pleinement signifié ; on est au cœur de la notion sémantique qu'il évoque. $N e ́$, préfixe d'un terme « discret » rend compte d'une collection d'éléments, alors que si le terme préfixé réfère à du « compact », l'idée d'ensemble est suggérée.

4. Les kavere n'ont jamais été des humains mais ils en ont l'apparence. De caractère imprévisible, ils peuvent aider ou perdre celui qui les rencontre.

5. La récolte des dèo doit être faite au moment de sa floraison, bunu wa viö (ö) (litt., fleur — faire fauciller, floraison qui appelle la faucille). Maurice Leenhardt signale aussi que ses informateurs, ex-anthropophages, disaient que c'était la période où les hommes étaient gras. 
vaient se consacrer à la construction ou à la réfection des cases ${ }^{6}$.

La «paille» était autrefois faucillée, öö, par les femmes à l'aide d'une valve d'huître perlière soigneusement polie et affûtée. Cet outil $\ddot{o} \ddot{\text {, du }}$ même nom que la technique, était percé de deux trous où passait une cordelette de poils de roussette (le dèré, un bien précieux) et faisait partie des trésors féminins. Réunies et liées en bottes, nérùrù, les graminées passaient aux mains des hommes et fournissaient les couvertures des cases.

La classification autochtone permet de distinguer des sous-espèces non répertoriées par les botanistes : dèo nawara et dèo jâ mie (litt. herbe - monnaie). Cette dernière, réputée comme la plus résistante, est donc dö dèo «la vraie». En effet, la vision organisatrice du monde a classifié toutes choses en deux systèmes binaires complémentaires : d'une part, ce qui est $X$ et le vrai $X$, d'autre part, ce qui est $X$ mâle et ce qui est $\mathrm{X}$ femelle. Les dèo bwè' (bwè' signifie «femme ») couvrent régulièrement le sol d'un tapis continu, tandis que les dèo wi' (wi' signifie " homme, mâle ») poussent en grandes touffes isolées.

(Jâ) mémé, Themada gigantea (L.), Graminée, pouvant atteindre jusqu'à $1 \mathrm{~m} 80$, n'était pas coupée mais arrachée pour être utilisée avec ses racines. Il existait en effet deux procédés de couvertures de cases. Arii mwâ (mwâ «maison») consistait à poser les nérùrù, bottes de paille dèo, sur les voliges et à les y maintenir en couches serrées se chevauchant le plus étroitement possible à l'aide de lianes. Celles-ci, soigneusement choisies pour leur qualité, étaient écrasées et passées dans l'alêne $a^{7}$. Cette technique, le yiru, demandait moins de peine et de temps que celle appelée jârêre $\hat{e}^{8}$ mais donnait des couvertures qui duraient moins longtemps, une dizaine d'années environ contre plus de vingt ans de protection des cases assurée par l'utilisation des touffes de mémé, racines à l'extérieur. Les pieds de graminées étaient entrés de force entre la structure de bois et de lianes de la toiture en les tassant au poing $(j \hat{a}){ }^{9}$.

Cette technique était probablement entièrement exécutée par les hommes et c'est peut-être en son honneur que se dansait, par les hommes seuls, le mémê. Les femmes qui n'avaient pas encore eu d'enfants ne devaient pas regarder cette danse et seules les « vieilles » étaient appelées à se joindre au cercle des danseurs à un moment donné ${ }^{10}$.

\section{Les nattes}

Le nattage jarè, tant par son nom que par le résultat obtenu, est évidemment à rapprocher de jârêrê. Jarè était le nom et de la technique et des nattes (couvertures ou capes) ainsi produites. La fabrication différait du nattage (lë) de brins de pandanus (kere karè), permettant d'obtenir des nattes lisses et serrées (amë). Dans le travail du jarè, l'ouvrière laissait de longues fibres libres créant ainsi une fourrure végétale, très efficace contre le froid, disait-on ${ }^{11}$. La graminée $j a ̂ u(u)$ (jëu à Poya), Eragrostis pilosa (L.) Beauv., Graminée ainsi que ses variétés jauu' rhiwa (litt. jauu' à divination) et jâ nédé' ou jâ xè nédé' (litt. herbe de l'inondation) étaient utilisées pour la confection de ce type de nattes.

Maurice Leenhardt signale que jâxûrû wi' (Paspalum orbiculare Forst., L., Graminée) était autrefois replantée et cultivée pour ses fibres. En revanche, jâxûrû bwè', sa femelle selon la classification locale, est une cypéracée, Scirpus sp. dont l'utilisation - nattage très serré pour obtenir une sorte de plat creux allongé pour mettre la nourriture - explique qu'elle se trouve rangée parmi les graminées. C'est, à notre connaissance, la seule « herbe » non graminée à rentrer dans la catégorie indiquée par $j a \hat{a}$, tandis que jinu ou jénu, qui est une graminée, ne s'y trouve pas. Jinu (Eleusine indica L. Gaertn., Graminée), est une grande touffe d'herbe qui pousse au bord des chemins et sur les rives des cours d'eau. Elle servait autrefois d'amarre aux pirogues et son

6. La construction ou la reprise d'une demeure abandonnée après une mort étaient liées aux cérémonies appelées bwêe, grandes manifestations sociales rassemblant des clans pour célébrer une fin de deuil, la « prise de bagayou » (étui pénien) des jeunes initiés, et servant à réaffirmer les liens et « chemins d'échange » des femmes entre les familles.

7. Le mot arii est décomposable en $a$ « alène» + rii, suffixe qui renvoie à l'accomplissement de la notion, ici, « se servir de l'alène ». Modernisée, la technique de couverture en paille est encore parfois utilisée, bien que les tôles aient généralement remplacé les chaumes.

8. Jârêrê est formé de jâ et du suffixe rêrê qui entre dans de nombreux termes techniques et signifie « se blesser à une pointe, blesser d'une pointe ». Ce fut le nom, au début de la colonisation, des bracelets de cuir hérissés de clous, armes de défense introduites probablement par les bagnards.

9. Dèo pouvait aussi être utilisée arrachée et non coupée mais était moins solide que mémé dont les bottes d'herbes arrachées repoussaient et créaient ainsi une toiture très résistante.

10. Bien peu de choses ont été notées sur ces danses " païennes » abandonnées ou interdites avec la christianisation. Lors de la construction de la grande case (case des hommes), les femmes, qui n'y seraient plus admises ensuite, étaient convoquées pour damer le sol en dansant.

11. Cette technique est totalement oubliée depuis longtemps. 
symbolisme d'ancrage lui a valu d'être un prénom féminin toujours employé.

\section{Les gazons}

C'est à un tout autre usage qu'était réservé le jâwia ou jawia (Chrysopogon aciculatus (Retz) Trin., Graminée), et son nom évoque encore aujourd'hui les gazons bien entretenus et uniformes. Autrefois, cette petite graminée couvrait d'un tapis dense les allées cérémonielles devant la grande case et, plus modestement, entourait toute demeure, pour y étendre les nattes où s'asseoir. Elle est encore plantée à cette fin mais est souvent remplacée par le «buffalo », raygrass importé, ou par jâève $(j a ̂+$ ramper + mentir, litt. l'herbe qui cherche à ramper), le chiendent local (Cynodon dactylon Pers., L., Graminée).

La ressemblance partielle avec une plante utile mais l'impossibilité de l'emploi d'une telle plante est à l'origine des appellations dépréciatives (en français, " ail de l'ours ", " oseille de crapaud », etc.), ainsi Apluda varia Hack, Graminée, est jawia i tibo (litt. le gazon de la déesse tibo) ${ }^{12}$ et une autre graminée Sporobolus virginicus (L.) Künth, jawia xùrù (litt. le gazon du pou).

\section{Le deuil, interdits et calendrier}

Cependant, l'herbe est aussi marque ${ }^{13}$. La poignée de graminées nouées interdit de récolter les fruits de l'arbre auquel elle est attachée, ou bien au milieu d'un sentier, accrochée sur un piquet, elle barre le passage vers le domaine abandonné d'un mort. Ainsi jâö, jâumé, jâmé sont des termes qui renvoient au deuil.

Jâö: «Herbe quelconque employée comme marque de la mort. Elle est attachée à l'aube du vingtième jour aux perches appelées wesara sur l'emplacement de la case du défunt (détruite après la mort) pour interdire l'accès du boeamoa. Employée aussi dans la divination » (Leenhardt, 1935 : 112). Malgré l'évolution des pratiques concernant le traitement des morts et le deuil, le terme reste le symbole des interdits et des dangers du monde invisible. De l'utilisation des graminées dans la divination, rien n'est connu, mais le nom des devins (il y en a toujours en activité) est jaa'uu ou jaa'oo. Le a n'est pas nasalisé. S'agit-il d'une rencontre ou d'une dérivation?

Jâumé désigne toujours le sol recouvert par plusieurs couches successives d'herbes dans les jachères qu'impose l'abandon des terres cultivées par le défunt. Le temps d'abandon est actuellement considérablement raccourci. Il était autrefois lié au bwêêjâumé et bwêê lélnéjâumé (litt. fête des jachères et fête de la fin des jachères, dernière fête offerte au mort) et ces fêtes devaient (théoriquement au moins) être prévues au minimum cinq ans après la mort de celui qu'on honorait. La jachère pouvait durer jusqu'à dix, quinze ans.

Le terme jâmé (litt. jâ + mort) désigne le veuf ou la veuve.

L'herbe, parce qu'elle se noue et forme ainsi une boule que l'on peut égrener sur un fil, sert à compter. Le nœud évoque la forme d'un fruit rond et jâ pwê kwé (litt. jâ+fruit+corde) désignait l'ancien calendrier ou la tenue des comptes. Jâ pwê kwé'i, c'était espacer les nœuds sur une cordelette pour y faire le comptage des lunes (varui signifie « lune, mois ») ou des dons de tas d'ignames reçus et donc à « rendre » le moment venu ${ }^{14}$.

\section{Le «statut » des jâ}

$J a ̂-$, la graminée, était une plante constamment présente dans la vie des femmes ${ }^{15}$, tant son utilisation journalière, comme lien de portage des charges, que pour le nattage, occupation féminine essentielle car les nattes étaient indispensables pour la vie quotidienne, les dons, les linceuls... Mais les hommes utilisaient aussi la graminée comme signe et symbole et elle concernait les deux sexes lors des travaux successifs pour la couverture des maisons. Le jâumé, domaine abandonné du défunt, était redoutable également pour tous. Ainsi, contrairement aux plantes « nobles » et en dépit d'une caractérisation sur des variétés, les graminées ne sont ni plantes mâles, comme les ignames, ni plantes femelles, comme les taros.

\section{Signification du terme jâ}

Bien qu'ils puissent souvent être utilisés indifféremment l'un à la place de l'autre, $j \hat{a}$ et $p \ddot{o}(i)$

12. La tibo est une déesse aux longs seins avec lesquels elle peut assommer les hommes qu'elle poursuit.

13. L'utilisation de l'herbe nouée en signe d'interdit se retrouve chez nous, généralement comme interdit de chasser sur la terre ainsi signalée.

14. En fait, il convient de rendre davantage que ce que l'on a reçu, mais sans humilier le récipiendaire et dans un laps de temps correct. Avoir oublié la quantité de ce qui a été reçu, c'est perdre la face.

15. Ce ne sont pas les herbes nouées qui signalait l'interdit qu'imposaient les règles de l'épouse — puisque l'herbe signifiait la mort — mais une feuille sèche de bananier suspendue devant sa case personnelle. 
correspondent à des actions distinctes. Jâ est nouer la poignée d'herbe sur elle-même, coinçant ou non dans ce nœud un objet, tandis que pöi, c'est attacher, lier des choses l'une à l'autre. C'est d'ailleurs pourquoi (pèvi) pö et pöi désignent les dons et les discours qui « rattachent» l'une à l'autre les familles lors des deuils.

Le terme $j \hat{a}$, au sens de nouer, lier, échappe évidemment au seul domaine de l'herbe. Ainsi jâvôrô était l'ensemble des lianes, des roseaux et des solives qui constituaient les « plafonds » des cases. Jâ vè nisyè (litt. jâ + dans le but de + faire pendre) est munir un paquet de nourriture d'un lien noué pour le suspendre afin de le protéger des rats, tandis que jâwî s'applique aux objets liés entre eux par des fibres. Ainsi é jâwînu, c'est lier deux éléments de feuilles de cocotier tressées pour en faire un écran. Cette technique était utilisée pour faire des barrages temporaires dans les « creeks " pour y pêcher à sec ${ }^{16}$, et le barrage et la pêche faite dans cette portion de rivière s'appelaient respectivement jấ' et vijâ.

Il est tentant de chercher une étymologie pour ce terme $j \hat{a}$ car il se retrouve sous la forme courte ou longue en composition dans de nombreux mots. L'étude de ces composés montre que $j \hat{a}-$ s'applique à tout ce qui est produit « spontané » de quelque chose. Or l'herbe semble pousser spontanément de la terre. Les Latins voyaient les abeilles sortir des entrailles d'une génisse ou d'un lion abattus, les Classiques pensaient que du linge abandonné dans un coin engendrait des souris, les gens de Houailou ont la même vision : les êtres, les choses exsudent une matière sui generis. Ainsi (né)jâ-kwijèi est la main de banane (kwijèi signifie "banane, bananier »); jâ(â)—é est « sa » vermine (pour l'homme, l'animal ou la plante) ${ }^{17}$; jââ-è est « sa » viscosité, par exemple la matière gluante qui protège les anguilles, les holothuries ou certains poissons; jâ-köö ( $k \ddot{o o ̈}$ « ciel ») est un petit nuage isolé dans un ciel bleu ; jâ-kuruö (kuruö « alizé qui souffle au début de la saison où verdit l'igname ») est le printemps ; jäwê (wê « trou d'eau ») est un petit crabe plat d'eau douce que les femmes pêchaient autrefois ; jâ-pèya" (pèya' signifie " pierre ») est une petite crevette qui vit sous les pierres ${ }^{18}$.
Mais c'est sous la forme jârâ (forme indépendante puisqu'elle est déterminée par son suffixe) que l'on trouve une liste toujours ouverte de termes que l'on peut traduire par « liquide produit par» : jârâ ji (produit de la tétée, de la succion) ou jârâ mèyâ (liquide du sein) « lait de la femme »; jârâ kwa" "goutte de pluie »; jârâ pièmè «larme» produite par l'œil qui coule ; jârâ dörö «miel d'abeille»; jârâ kê «sève d'arbre »; jârâ nu " lait de coco »; jârâ mwa, mёu, maniö «suc de taro, d'igname, de manioc $» 19$.

\section{Conclusion}

Plantes non cultivées donc plantes sans but précis, les graminées ont été cependant soigneusement répertoriées pour les divers usages qu'on en pouvait faire. Quotidiennement utilisées (liens de toutes sortes, nattes, chaumes, etc.), elles accompagnaient la vie des femmes et des hommes de la naissance (courroies de portage des berceaux-plateaux) à la mort (nattes, linceuls, interdits des jachères et calendriers des rites funéraires...) et même dans le monde invisible (divination), sans qu'elles soient jamais devenues plantes vivrières comme ce fut ailleurs le cas pour les céréales.

\section{RÉFÉRENCES}

BARRAU, J., 1956. L'agriculture vivrière autochtone de la Nouvelle-Calédonie. Nouméa : Commission du Pacifique Sud.

Aramiou Sylvain, Jean EuriteIn et Georges Kavivioro, nd [2001]. Dictionnaire a'jië-français, Nouméa, Fédération de l'enseignement libre protestant (FELP).

La Fontinelle, J. de, 1972. Sexes, plantes et éléments à Houailou. in J. M.C. Thomas et L. Bernot (eds), Langues et Techniques, Nature et Société. Paris: Klincksieck, vol. 1, pp. $283-293$.

LEENHARDT, M., 1935. Vocabulaire et Grammaire de la langue Houailou. Paris : Institut d'Ethnologie.

16. Les droits de pêche qui existaient sur les rivières comme sur les rivages marins étaient rigoureusement respectés et l'utilisation de ces lieux contrôlée afin de ne pas dépeupler les eaux. Ce n'est que lors des grandes fêtes, les bwêe, pendant lesquelles il fallait nourrir un grand nombre d'invités durant plusieurs jours, que l'on avait recours à cette technique d'assèchement.

17. Il se fait aujourd'hui une confusion entre $j \hat{a}$ — « produit de » et ja' ou jaa qui désignaient le ver de pourriture, les chenilles, ceci au travers d'un chevauchement de sens, le jaa mwa', la chenille qui vit sur les taros, étant vue comme sa vermine.

18. Peut-être faudrait-il ajouter ici les roches noires, calcaires ruiniformes qui se dressent isolées dans le paysage. Appelées $j \hat{a}$, elles sont objets de croyances et hérö̈nes de récits.

19. Dans les discours traditionnels, le suc des ignames et le suc de taros mangés par les parents sont considérés engendrer les enfants. Le manioc est importé. 